\title{
A COMPACT MODULAR ARCHITECTURE FOR THE REALIZATION OF HIGH-SPEED BINARY SORTING ENGINES BASED ON RANK ORDERING
}

\author{
I. Hatırnaz, F. K. Gürkaynak and Y. Leblebici \\ Worcester Polytechnic Institute \\ Department of Electrical and Computer Engineering \\ Worcester, MA 01609-2280
}

\begin{abstract}
A new modular architecture is presented for the realization of high-speed binary sorting engines, based on efficient rank ordering. Capacitive Threshold Logic (CTL) gates are utilized for the implementation of the multi-input programmable majority (voting) functions required in the architecture. The overall complexity of the proposed bitserial architecture increases linearly with the number of input vectors to be sorted (window size $=\mathrm{m}$ ) and with the bitlength of the input vectors (word size $=n$ ), and the sorter architecture can be easily expanded to accommodate large vector sets. Detailed simulations indicate that the sorter structure can operate at sampling clock rates of up to 50 $\mathrm{MHz}$, where the throughput is boosted by fine-grain pipelining. It is demonstrated that the proposed sorting engine is capable of producing a fully sorted output vector set in $(m+n-1)$ clock cycles, i.e., in linear time.
\end{abstract}

\section{Introduction}

The task of sorting an arbitrarily ordered vector set according to magnitude (either from-largest-to-smallest or fromsmallest-to-largest) is one of the fundamental operations required in many digital signal processing applications. It is also an expensive operation in terms of area-time complexity; software-based solutions require word-level sorting and can become computationally intensive, while the overall complexity of hardware-based solutions usually increases very rapidly with the size of the input vector set (number of vectors) and with the bit-length of the input vectors [6], [1], [3]. The design of efficient sorting engine architectures is therefore a significant challenge for overcoming the computational bottleneck of the binary sorting problem. A number of recent proposals for the realization of sorting networks rely primarily on median or rank order filters (ROF), yet their capabilities in terms of window size and bit-length are typically limited due to rapidly increasing hardware complexity [6], [7], [2].

In this work, we present a compact and fully modular sorting engine architecture that is capable of processing a large number of input vectors in linear time. The overall architecture is completely scalable to accommodate a wide range of window sizes and bit-lengths, and the hardware complexity only grows linearly with both of these parameters. The proposed sorter architecture is essentially based on a fully programmable modular ROF design that was presented earlier [10], [11]. In the following, we first discuss the programmable ROF architecture that forms the basis of the sorting engine, in Section 2. The realization of the sorter is presented in Section 3, followed by conclusions in Section 4.

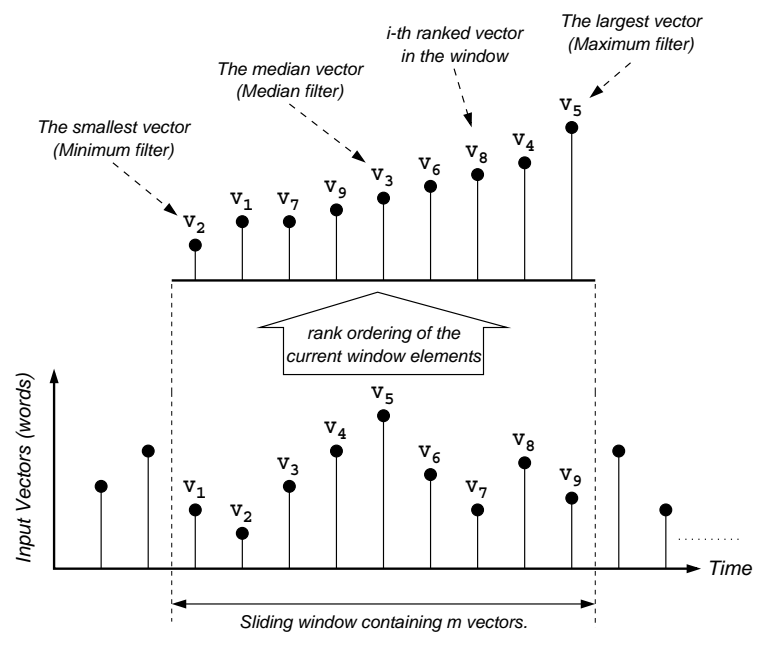

Figure 1: One-dimensional illustration of the rank-ordering process.

\section{The Programmable ROF Architecture}

The rank order filter (ROF) is a non-linear digital filter which determines the i-th ranking element in a given window consisting of binary encoded input words (Fig. 1). Special cases of rank order filters are median, minimum and maxi- 


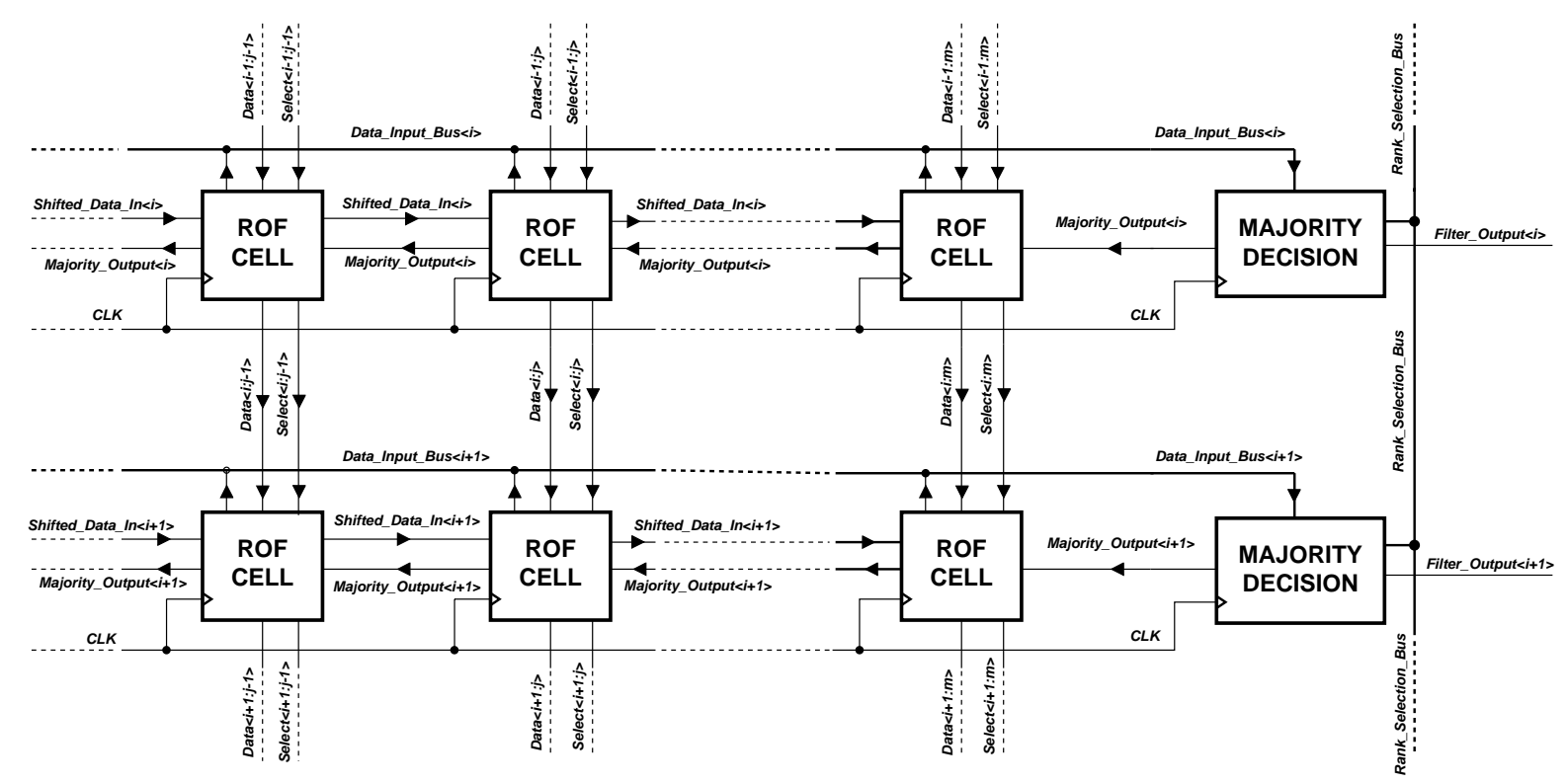

Figure 2: Detailed signal flow between modular ROF-cells and majority gates.

mum filters, where the outputs are the median, the minimum and the maximum values of the input words, respectively [1]. Variants of ROFs especially, median filters are widely used in digital signal and image/video processing because of their non-linear characteristics.

In recent years, some innovative bit-serial structures for rank-order filters have been presented, which are mostly based on majority-decision algorithms [4], [9]. Yet, the majority function is typically hard to realize using conventional Boolean building blocks, since it requires a large number of gates and a large logic depth.

\subsection{The Rank Ordering Algorithm}

A bit-serial algorithm first proposed in [6] was chosen as the basis of the programmable rank-order filter architecture implemented in this work. In this algorithm, the problem of finding a rank-order-selection for n-bit long words is reduced to finding " $\mathrm{n}$ " rank-order-selections for 1-bit numbers.

The algorithm starts by processing the most significant bits (MSB) of the $m=(2 \mathrm{~N}+1)$ words in the current window, through an m-input programmable majority gate, to yield the MSB of the desired filter output. This output is then compared with the other MSBs of the window elements. The vectors whose MSB is not equal to the filter output have their MSB propagated down by one position, replacing the less significant bits of the corresponding words.

The bit-serial operation flow of the algorithm described above suggests a very simple bit-level pipelined data path architecture, consisting of data modifier-propagator blocks to handle fine-grained data selection, and majority decision blocks to determine output bits.

Identical 1-bit filter slices can be used in sequence (cascade configuration) in order to process input vectors of arbitrary bit-length. The modular structure of the one-bit slice described above also allows for scalable realization of the ROFs with different window sizes and word lengths. Details of the realization of this rank ordering algorithm were presented earlier in [10] and [11].

\subsection{Implementation of the Programmable ROF Architecture}

A programmable rank-order filter of any window size and word-length can be realized by using the two main blocks described above. The word-length dictates the number of the majority decision gates, whereas the window size determines the number of ROF-cells driving one of these majority gates. The programmable majority decision gates are realized using the capacitive threshold logic (CTL) circuit architecture presented earlier [5]. This allows simple implementation of programmable majority gates with up to 63 parallel inputs, using a very small silicon area $(625 \mu \mathrm{m} \times$ $130 \mu \mathrm{m}$ for 63-bit majority gate).

The signal flow between the ROF cells and the majority gates are shown in Figure 2. The modular architecture consisting of only two major blocks enables fully scalable construction of filter structures of arbitrary size. This structure operates with a latency of (n-1) clock cycles, producing one binary output vector (corresponding to the $\mathrm{k}^{\text {th }}$ ranked vector in the input window) in each clock cycle. 


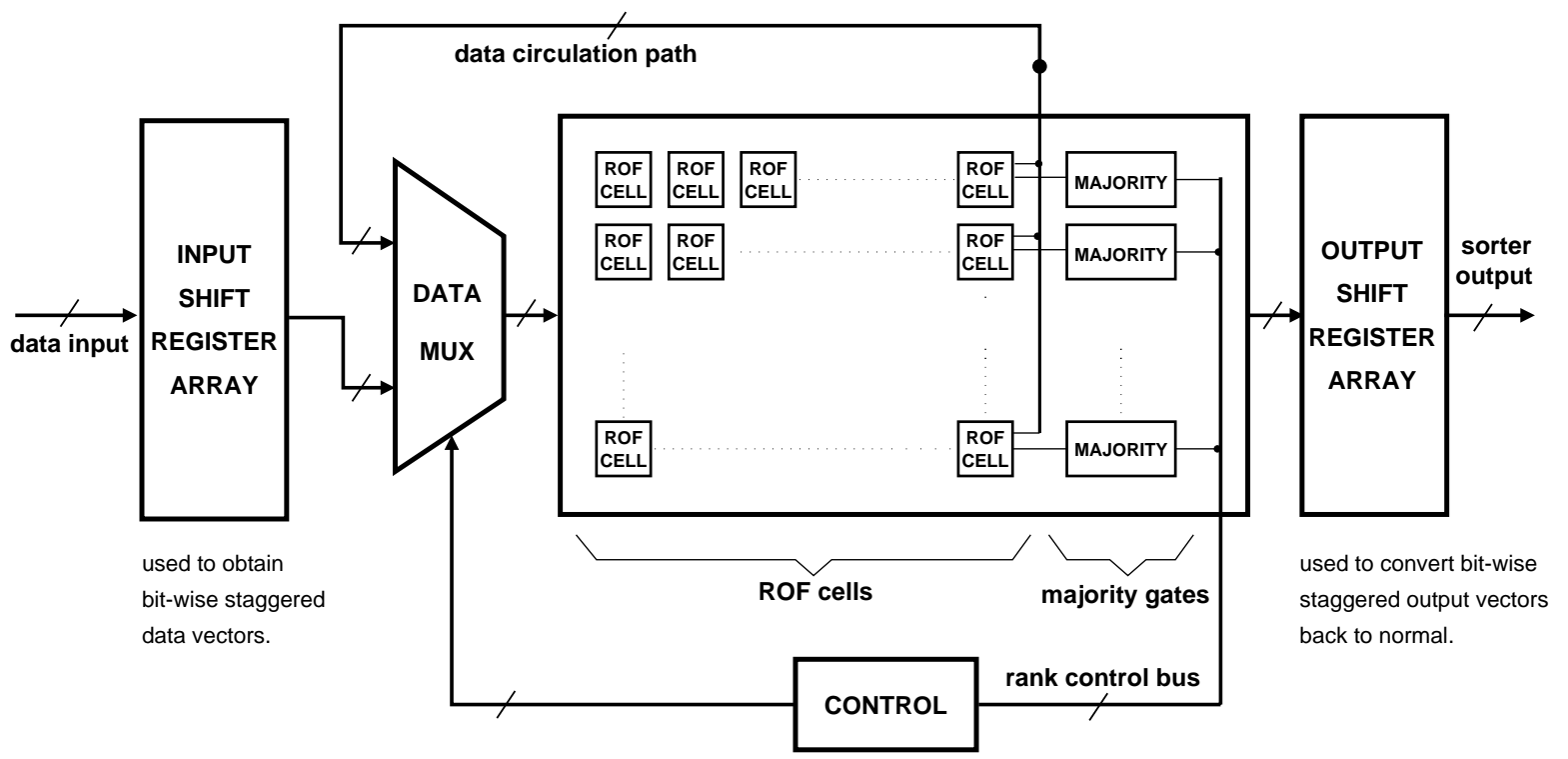

Figure 3: Overall architecture of the proposed sorter engine.

\section{Realization of the Sorting Engine}

The proposed sorter architecture exploits the fact that the modular ROF core described in Section 2 is capable of generating one output vector per clock cycle, corresponding to the currently selected rank. If the ranking process is repeated on the same set of vectors instead of processing a continuous stream of new vectors, the members of the vector set can be sorted in linear time by simply changing (increasing or decreasing) the rank in each clock cycle. The overall architecture of the sorting engine is shown in Figure 3. The flow of data through the modular ROF core is being regulated by complementary input and output shift registers, which are used to stagger the individual bit-planes of each input vector to enable bit-level pipelined operation. The multiplexer on the input side is used for accepting the input vectors at the rate of one vector per clock cycle, as well as for circulating (rotating) the data until sorting is completed. The control logic is responsible for regulating the data circulation path, and for applying the rank selection signals to the individual bit-planes, in ascending or descending order. The fact that each individual bit-plane is capable of processing a different rank at any given time significantly increases the overall efficiency of this architecture. In a typical sorting run, the control logic simply requests each bit-plane to process a different rank in each clock cycle, either beginning from the maximum rank and descending, or beginning from the minimum rank and ascending.

The operation of the proposed sorting engine is illustrated with an example in Figure 4. Here, five 4-bit vectors (A through E) are being sorted by the ROF core. Note that the first rank (R1) is initially applied to the MSB plane con- sisting of the bits A1 through E1. In the next clock cycle, the same rank is used to process the lesser-significant bit-plane (A2 through E2), while a new rank (R2) is being applied to the MSB plane. Also note that the staggered data bits are gradually circulated from the end of the chain to the front, so that each vector in the window can be completely processed. The entire operation requires only $(m+n-1)$ clock cycles after all input vectors are applied. It is important to note that the time-complexity of the sorting operation described above has a linear dependence both with respect to window size (m) and with respect to word-length (n).

A complete VHDL model of the proposed sorter architecture has also been developed to verify its operation. The synthesized sorter architecture contains a total of 2190 NAND-equivalent gates, including the input and output shift registers, data multiplexer, and the ROF core. Note that each 8-bit majority function in this synthesized structure requires 130 NAND-equivalent gates, while each ROF cell has a logic complexity of about 14 NAND gates. Fig. 5 shows simulated results of two sorting operations on an arbitrarily ordered set of eight vectors, each with a word-length of 8 bits. It can be seen that the first output vector is generated with a latency of (n-1) clock cycles, after the last vector of the set is entered.

\section{Conclusion}

A modular architecture has been presented for the realization of high-speed binary sorting engines, based on an efficient rank ordering scheme. The overall complexity of the proposed bit-serial architecture increases linearly with the 


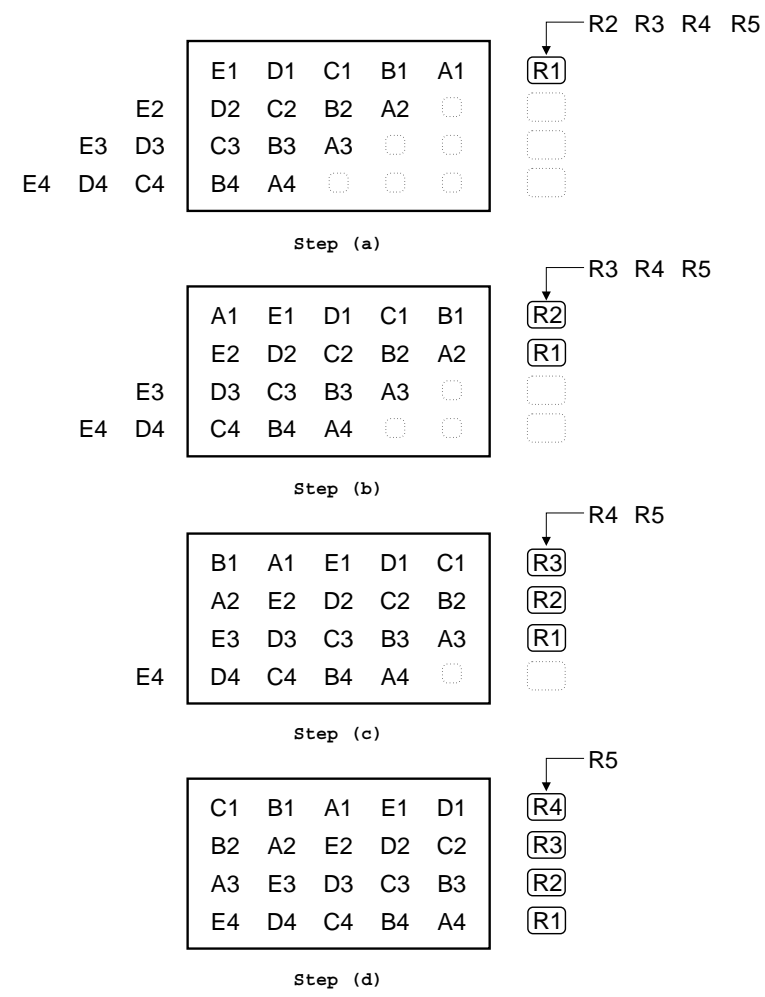

Figure 4: Illustration of a sorting operation on five 4-bit input vectors in four steps: (a) The staggered input vectors are shifted into the ROF core, and the first rank (R1) is applied to the MSB plane. (b) The MSB of the first input vector (A1) is rotated, R1 is applied to the next bit-plane, and the new rank R2 is applied to the MSB plane. (c) B1 and A2 are rotated, while R1 is applied to the lesser-significant bitplane. The rank R2 shifts down by one, while R3 is applied to the MSB plane. (d) Bit circulation (rotation) continues, while the ranks propagate down the bit-planes in descending order.

number of input vectors to be sorted and with the bit-length of the input vectors. It was demonstrated that the proposed sorting engine is capable of producing a fully sorted output vector set in $(m+n-1)$ clock cycles.

\section{REFERENCES}

[1] D.S. Richards, "VLSI median filters", IEEE Trans. Acoust., Speech, Signal Processing, vol. 38, pp.145-152, January, 1990.

[2] W.K. Lam and C.K. Li, "Binary sorter by majority gate", IEE Electronic Letters, Vol. 32, July 1996.

[3] P. Wendt et al., "Stack filters", IEEE Trans. Acoust., Speech, Signal Processing, pp. 898-911, 1986.

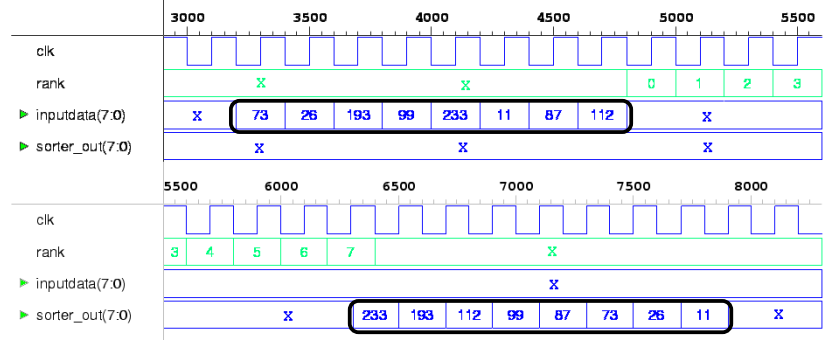

Figure 5: Simulation results of a ranking operation on an arbitrarily ordered set of eight vectors. The input set is being sorted in descending order from maximum (233) to minimum (11) value. The input set can also be sorted in ascending order from minimum to maximum value, simply by changing the rank sequence applied to the majority gates of each bit-plane.

[4] A. Gasteratos, I. Andreadis, Ph. Tsalides, "Realization of rank order filters based on majority gate", Pattern Recognition, vol.30, no. 9, pp 1571-1576, 1997.

[5] Y. Leblebici, F.K. Gurkaynak, D. Mlynek, "A compact 31-input programmable majority gate based on capacitive threshold logic", in Proc. IEEE Int. ASIC Conference 1998, pp. 281-285.

[6] B.K. Kar, D.K. Pradhan, "A new algorithm for order statistic and sorting", IEEE Trans. on Signal Processing, vol. 41, pp.2688-2694, August 1993.

[7] C.C. Lin, C.J. Kuo, "Fast response 2-D rank order algorithm by using max-min sorting network", International Conference on Image Processing 1996, Vol. 1, pp. 403-406.

[8] C. Chen, L. Chen, T. Chiueh, J. Hsiao, "An efficient pipelined VLSI implementation of rank order filter", ISSIPNN 1994, Vol. 2, pp. 630-633.

[9] C.L. Lee and C.W. Jen, "Bit-sliced median filter design based on majority gate", in Proc. Ins. Elec. Eng.-G, vol 139, pp.63-71, 1992.

[10] İ. Hatırnaz, F.K. Gurkaynak, Y. Leblebici, "Realization of a programmable rank-order filter architecture using capacitive threshold logic gates", ISCAS'99 Proceedings, 1999.

[11] İ. Hatırnaz, F.K. Gurkaynak, Y. Leblebici, "A modular and scalable architecture for the realization of high-speed programmable rank-order filters", ASIC/SOC'99 Proceedings, pp. 382-386, 1999. 